\title{
Numerical Simulations of a Two-dimensional Vertically Averaged Air Pollution Measurement in a Street Canyon
}

\author{
Hasakarn Thongzunhor ${ }^{1}$, Nopparat Pochai ${ }^{1,2, *}$ \\ ${ }^{1}$ Department of Mathematics, Faculty of Science, King Mongkut's Institute of Technology Ladkrabang, Bangkok, 10520, Thailand \\ ${ }^{2}$ Centre of Excellence in Mathematics CHE, Si Ayutthaya Rd. Bangkok, 10400, Thailand
}

Received June 16, 2021; Revised October 26, 2021; Accepted January 20, 2022

\begin{abstract}
Cite This Paper in the following Citation Styles
(a): [1] Hasakarn Thongzunhor, Nopparat Pochai , "Numerical Simulations of a Two-dimensional Vertically Averaged Air Pollution Measurement in a Street Canyon," Environment and Ecology Research, Vol. 10, No. 1, pp. 107 - 115, 2022. DOI: 10.13189/eer.2022.100110.
\end{abstract}

(b): Hasakarn Thongzunhor, Nopparat Pochai (2022). Numerical Simulations of a Two-dimensional Vertically Averaged Air Pollution Measurement in a Street Canyon. Environment and Ecology Research, 10(1), 107 - 115. DOI: 10.13189/eer.2022.100110.

Copyright $\odot 2022$ by authors, all rights reserved. Authors agree that this article remains permanently open access under the terms of the Creative Commons Attribution License 4.0 International License

\begin{abstract}
Air pollution is the release of pollutants into the atmosphere that are harmful to human health and the ecosystem as a whole. Initially, urban air pollution was considered to be a regional problem caused largely by domestic heating and industrial emissions, both of which are now well under control. The building's canyon structure and the geometry of the streets in urban areas are street canyons. Side Street connects the two sides of the street, which are made up of portions of buildings. Street canyons, which are urban streets bordered on both sides by structures, have shown high levels of pollution. Pedestrians, cyclists, vehicles, and residents will most likely be surrounded by pollution concentrations higher than current air quality limits on these walkways. The research is focused on detecting air pollution in a street canyon. There will be an introduction to a transient two-dimensional advection-diffusion equation. A two-dimensional vertically averaged air pollution measurement model is utilized to characterize the air pollution concentration along a street canyon. The model delivers the pollutant concentration in the air each and every time. The model's air pollutant concentration is approximated using a finite difference technique. An approximation approach to open and closed boundary conditions is proposed. Wind direction effects are also modelled. The suggested numerical approaches performed well in producing a high level of agreement. Simple explicit schemes have the benefit of being simple to compute. These techniques may be used to simulate air pollution measurements in a variety of street canyons.
\end{abstract}

Keywords Air Pollution Measurement, Street Canyon, Vertically Averaged, Two-dimensional, Numerical Simulations

\section{Introduction}

Air contamination has been a critical natural and wellbeing concern for hundreds of years. This presentation is across the board and is vital for all citizens since it is unavoidable. The Global Burden of Disease 2010 assessed that 3.1,3.5, and 0.2 million deaths happened every year as an after effect of exposures to encompassing particulate matter, strong energizes, and surrounding ozone contamination, separately. With the fast urbanization of the total populace, air quality is expected to be on the decline as a wellspring of contamination in total. In profoundly populated urban areas, daily life and human activities (e.g., controlling era and vehicle usage) must ascend to stay aware of the requests of developing areas.

Initially, urban air pollution was thought to be a local issue caused mostly by residential heating and industrial pollutants, both of which are now largely over control. The poisons related to the primary activity are $\mathrm{CO}, \mathrm{NO}_{x}$, hydrocarbons, and particles. $C O$ is a defective fuel for ignition. Burning additionally delivers a blend of $\mathrm{NO}_{2}$ and $N O$, of which more than $90 \%$ compared to $N O$. An extensive variety of unburned and artificially changed 
hydrocarbons (e.g., benzene, toluene, ethane, ethylene, pentane, and so forth) is discharged by engine vehicles through various diverse procedures (e.g., dissipation, fuel tank removal, oil leak, etc.). Finally, particles of dense carbonaceous material are transmitted for the most part by diesel and inadequately kept up by petrol vehicles.

The air pollution in two dimensional spaces with obstacles domain is also studied [2], [5]. The three-dimensional fractional step method is applied based on the discretization of the time dependent atmosphere advection-diffusion equation [4]. The air pollution problem in three dimensional spaces with multiple sources is presented. The initial conditions in the domain are assumed to be zero everywhere without obstacles [6]. They use the finite difference method in the air pollution model of two-dimensional spaces with single - point source [3], a semi-empirical mathematical model, Urban Street Model (USM) to efficiently estimate the dispersion of vehicular air pollution in cities. This model combines building density, building heights, and the permeability of building arrangements in relation to wind flow to represent urban building configurations (Olga et al., 2009). The model estimates $\mathrm{NO}_{2}$ concentrations using a simple photochemistry scheme. SEUS is applied to estimate $N O_{x}$ and $\mathrm{NO}_{2}$ hourly concentrations in an irregular and busy street canyon in the city of Buenos Aires [7]. Air quality affected by trees in real street canyons: The case of Marylebone neighborhood in central London [1].

Human exposure to dangerous chemicals is expected to grow substantially in urban areas, particularly in areas with a fairly high population and activity. This is frequently the case around high activity areas in downtown regions, where urban geology and microclimate can contribute towards the development of poor air scattering conditions that allow pollution hotspots to grow. High contamination levels have been seen in street canyons, which are urban walkways bordered on both sides by structures. Walkers, bikers, vehicles, and residents will most likely be surrounded by pollution concentrations that surpass existing air quality regulations inside these walkways.

According to their related articles [1]-[7], we are also considering air pollution measurement in a street canyon as well. In this research, the air pollution measurement in a street canyon is the focus. A transient two-dimensional advection-diffusion equation will be introduced to describe the simulation. A two-dimensional vertically averaged air pollution measurement model is used to describe the air pollutant concentration along a street canyon. The model provides the air pollutant concentration each and every time. A finite difference technique is used to approximate the air pollutant concentration of the model. There is air pollution in the street canyon, which is driven by the pollutants around the boundary of the area. An approximation technique for open boundary conditions and closed boundary conditions is proposed. The effect of wind directions is also simulated.

\section{Governing equation}

\subsection{Street canyon domain}

The canyon structure of buildings and streets in urban settings is known as urban street canyon. As seen in Fig. 1 and Fig. 2, the sides of the street are made up of chunks of buildings, with side streets in between. In this study, we assume that there is no cross-street airflow. Consequently, there is no air flow direction as shown in Fig.1. The contaminants might come from vehicles entering within the canyon. Other hazardous sources might be found at the canyon's street entrance or on one of the canyon's side streets. Cars and vehicles produce mobile sources of air pollutants in the street canyon. Combustion and fuel evaporation from mobile sources contaminate the air. These emissions contribute substantially to air pollution, including air toxics and greenhouse gases.

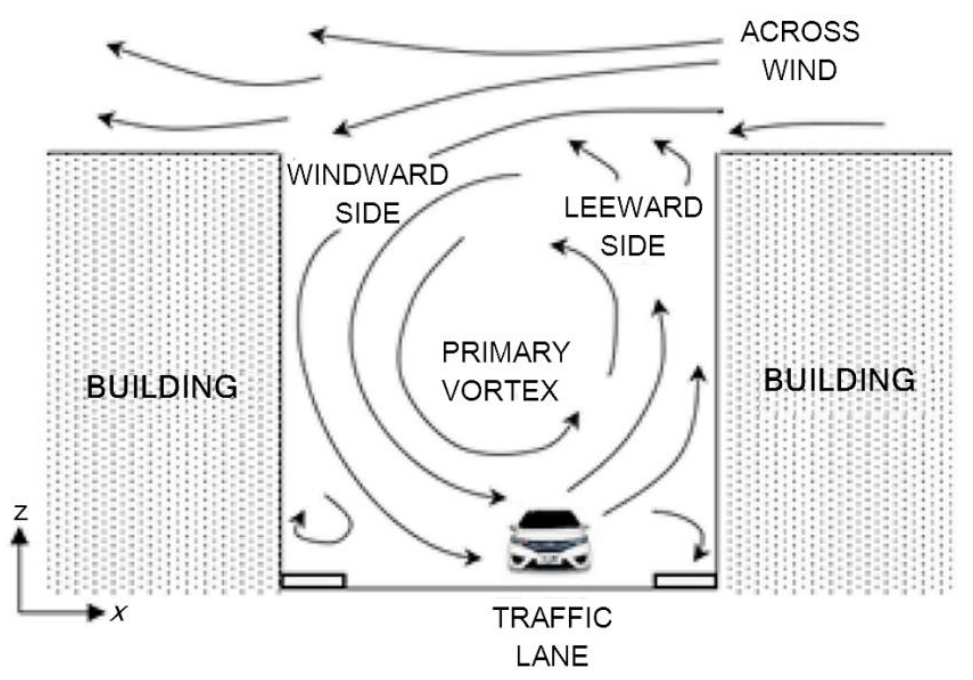

Figure 1. Urban street canyon and Schematic of cross-street air circulation in a street canyon 


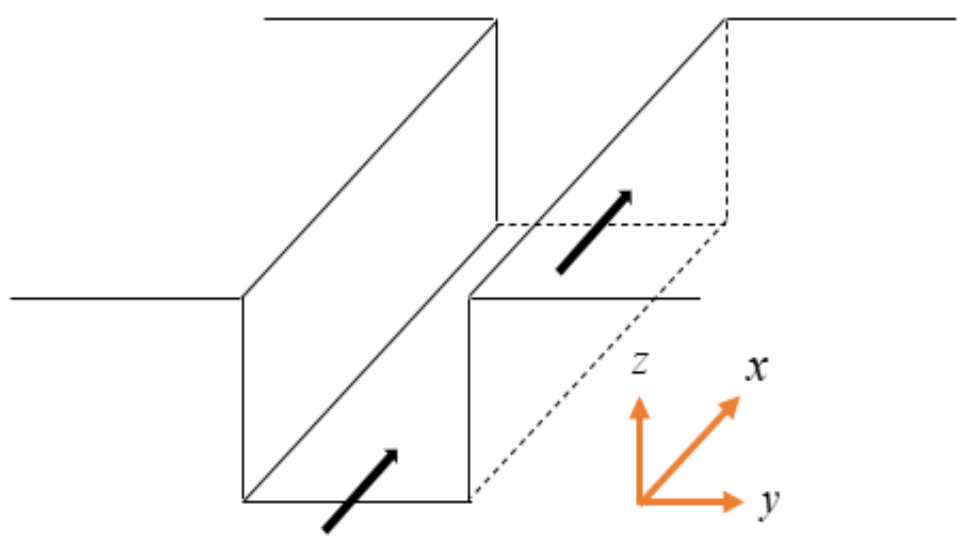

Figure 2. Three-dimensional street canyon domain

\subsection{Governing Equation}

\subsubsection{Air Pollution Dispersion Model}

Assume that there is no cross-street airflow in a street canyon, as shown in Fig.2. The dispersed air pollutant process satisfies a mass transfer equation that involves transmission and diffusion. We obtain a two-dimensional vertically averaged advection-diffusion equation by averaging the considered domain across the lateral dimension,

$$
\frac{\partial c}{\partial t}+u \frac{\partial c}{\partial x}+v \frac{\partial c}{\partial y}=D_{x y}\left(\frac{\partial^{2} c}{\partial x^{2}}+\frac{\partial^{2} c}{\partial y^{2}}\right)+Q,
$$

for all $\Omega=\{(x, y, t) ; 0 \leq x \leq L, 0 \leq y \leq W, 0 \leq t \leq T\}$ where $L$ is the length of the considered street canyon, $W$ is the width of the considered street canyon, $T$ is the end of a simulation time, $c(x, \mathrm{y}, t)$ is the vertically averaged air pollutant concentration at the point $(x, y)$ at time $t, u$ is a constant wind speed in the $\mathrm{x}$ direction, $v$ is a constant wind speed in the y-direction, $D_{x y}$ is the diffusion coefficient of the considered air pollutant matter in $\mathrm{x}$ and $\mathrm{y}$ direction, $Q(x, \mathrm{y}, t)$ is the rate of change of concentration in the street canyon per unit of time due to the vehicle moving as a mobile source around the street canyon.

\subsubsection{Initial and Boundary Conditions}

(a) The initial condition

The potential air pollutant concentration in the considered canyon is described by

$$
c(x, y, 0)=f(x, y) \text { for all }(x, y) \in[0, L] \times[0, W] \text {. }
$$

(b) The boundary conditions

The considered domain boundary can be classified into 3 types as shown in Fig.3 as below,

I. The measured boundary is described by $c(x, y, t)=c_{0} \quad$ where $\quad x=0$ for all $y \in[0, W], t \in[0, T], \quad c_{0}$ is the measured air pollutant concentration on the canyon entrance.

II. The fully-open boundary is described by $\left.\frac{\partial c}{\partial y}\right|_{(x, y, t)}=0$, where $y=0$ or $y=W$ for all $x \in[0, L], t \in[0, T]$.

III. The opened boundary is described by $\left.\frac{\partial c}{\partial x}\right|_{(x, y, t)}=\kappa$, where $x=0$ or $x=L$ for all $y \in[0, W], t \in[0, T], \kappa$ is the rate of change of air pollutant concentration with respect to $\mathrm{X}$-direction. 


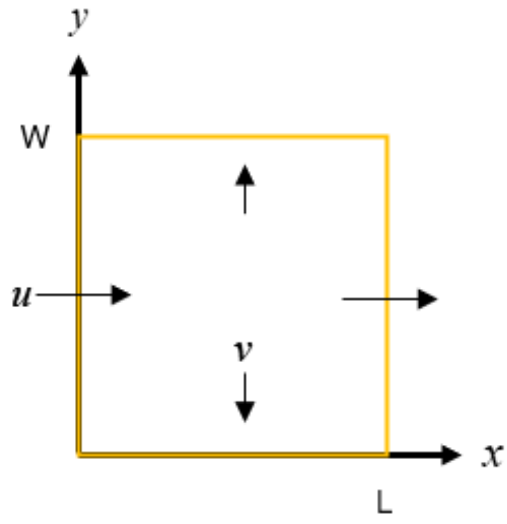

(a)

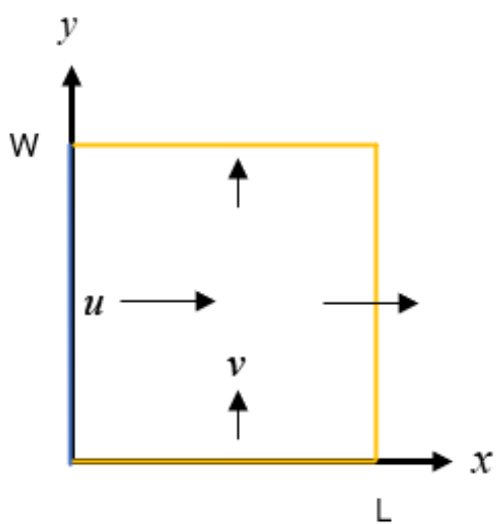

(b)

Figure 3. (a) The wind direction for $\mathrm{x}$ - and $\mathrm{y}$-direction for simulation 1; (b) The wind direction for $\mathrm{x}$ - and $\mathrm{y}$-direction for simulations 2 and 3

\section{Numerical Techniques}

In the advection-diffusion equation, the forward difference in time and the center difference in space are used Eq.(2.1). We can approximate $c(x, y, t)=c_{i, j}^{n}$ are the values difference approximation of at point $x=i \Delta x, y=j \Delta y \quad$ and $\quad t=n \Delta t \quad$ where $0 \leq i \leq L, 0 \leq j \leq W$ and $0 \leq n \leq N$. The grid point $c\left(x_{i}, y_{j}, t_{n}\right)$ is defined by $x_{i}=i \Delta x$ for all $i=0,1,2, \ldots, M$ and $y_{j}=j \Delta y$ for all $j=0,1,2, \ldots, P$ and $t_{n}=n \Delta t$ for all $n=0,1,2, \ldots, N$ in which $M, P$ and $N$ are positive integers. Using the forward time center space method to Eq. (2.1), the following finite difference equation can be obtained:

$$
\begin{aligned}
& c \cong c_{i, j}^{n} \\
& \frac{\partial c}{\partial t} \cong \frac{c_{i, j}^{n+1}-c_{i, j}^{n}}{\Delta t} \\
& \frac{\partial c}{\partial x} \cong \frac{c_{i+1, j}^{n}-c_{i-1, j}^{n}}{2 \Delta x} \\
& \frac{\partial c}{\partial y} \cong \frac{c_{i, j+1}^{n}-c_{i, j-1}^{n}}{2 \Delta y} \\
& \frac{\partial^{2} c}{\partial x^{2}} \cong \frac{c_{i+1, j}^{n}-2 c_{i, j}^{n}+c_{i-1, j}^{n}}{(\Delta x)^{2}} \\
& \frac{\partial^{2} c}{\partial y^{2}} \cong \frac{c_{i, j+1}^{n}-2 c_{i, j}^{n}+c_{i, j-1}^{n}}{(\Delta y)^{2}} \\
& Q \cong Q_{i, j}^{n}
\end{aligned}
$$

substituting Eq.(3.1-3.7) into Eq. (2.1), we can obtain an explicit form of finite difference equation as follows,

$$
c_{i, j}^{n+1}=B_{1} c_{i-1, j}^{n}+B_{2} c_{i, j-1}^{n}+B_{3} c_{i, j}^{n}+B_{4} c_{i, j+1}^{n}+B_{5} c_{i+1, j}^{n}+Q_{i, j}^{n}
$$

where

$$
\begin{gathered}
B_{1}=D_{x y} \frac{\Delta t}{(\Delta x)^{2}}+u \frac{\Delta t}{2 \Delta x}, \\
B_{2}=D_{x y} \frac{\Delta t}{(\Delta y)^{2}}+v \frac{\Delta t}{2 \Delta y}, \\
B_{3}=1-2 D_{x y} \frac{\Delta t}{(\Delta x)^{2}}-2 D_{x y} \frac{\Delta t}{(\Delta y)^{2}}, \\
B_{4}=D_{x y} \frac{\Delta t}{(\Delta y)^{2}}-v \frac{\Delta t}{2 \Delta y}, \\
B_{5}=D_{x y} \frac{\Delta t}{(\Delta x)^{2}}-u \frac{\Delta t}{2 \Delta x} .
\end{gathered}
$$

If $c_{i, j}^{n}$ are lay at the boundary of the domain, it was calculated by applying the backward different schemes at right boundary and top boundary, forward different schemes at left boundary and ground boundary.

Case I, left boundary condition, where $i=0$ and $0 \leq j \leq W$, substituting the approximate unknown vector nodes $c_{-1, j}^{n}$ of left boundary into Eq. (3.8), we had

$$
\frac{\partial c}{\partial x}(0, y, t)=c_{1} \text { or } \frac{\partial c_{0, j}^{n}}{\partial x}=c_{1}
$$

using backward difference,

$$
\begin{gathered}
\frac{c_{0, j}^{n}-c_{-1, j}^{n}}{\Delta x}=c_{1} \\
c_{-1, j}^{n}=c_{0, j}^{n}-c_{1} \Delta x \quad j=0,1,2, \ldots, P
\end{gathered}
$$

Case II, right boundary condition, where $i=L$ and 
$0 \leq j \leq W$, substituting the approximate unknown vector nodes $c_{L+1, j}^{n}$ of right boundary into Eq. (3.8), we had

$$
\frac{\partial c}{\partial x}(L, y, t)=c_{1} \text { or } \frac{\partial c_{L, j}^{n}}{\partial x}=c_{1}
$$

using forward difference,

$$
\begin{gathered}
\frac{c_{L+1, j}^{n}-c_{L, j}^{n}}{\Delta x}=c_{2} \\
c_{L+1, j}^{n}=c_{0, j}^{n}+c_{2} \Delta x \quad j=0,1,2, \ldots, P
\end{gathered}
$$

Case III, ground boundary condition, where $0 \leq i \leq L$ and $j=0$, substituting the approximate unknown vector nodes $c_{i,-1}^{n}$ of ground boundary into Eq.(3.8), we had

$$
\frac{\partial c}{\partial y}(y, 0, t)=c_{3} \text { or } \frac{\partial c_{i, 0}^{n}}{\partial y}=c_{3}
$$

using backward difference,

$$
\begin{gathered}
\frac{c_{i, 0}^{n}-c_{i,-1}^{n}}{\Delta y}=c_{3} \\
c_{i,-1}^{n}=c_{i, 0}^{n}-c_{3} \Delta y \quad i=0,1,2, \ldots, M
\end{gathered}
$$

Case IV, top boundary condition, where $0 \leq i \leq L$ and $j=W$, substituting the approximate unknown vector nodes $c_{i, W+1}^{n}$ of top boundary into Eq.(3.8), we had

$$
\frac{\partial c}{\partial y}(x, W, t)=c_{4} \text { or } \frac{\partial c_{i, W}^{n}}{\partial y}=c_{4}
$$

using forward difference,

$$
\begin{gathered}
\frac{c_{i, W+1}^{n}-c_{i, W}^{n}}{\Delta y}=c_{4} \\
c_{i, W+1}^{n}=c_{i, W}^{n}+c_{4} \Delta y \quad i=0,1,2, \ldots, M
\end{gathered}
$$

\section{Numerical Experiments}

In this section we present four examples. We consider the canyon is square in shape, and so consider in domain $0 \leq x \leq 1$ and $0 \leq y \leq 1$, with initial and boundary conditions as set out below for each particular example.

\subsection{Simulation 1}

Smoothly air flow along a street canyon. Assuming that the averaged air flow velocity in $\mathrm{x}$-and $\mathrm{y}$-direction are constants, $u(x, y, t)=0.1$, for all

$(x, y, t) \in \Omega, \quad v(x, y, t)=v_{1}=-0.1$ for all $x \in[0,0.5]$, $y \in[0,1], t \in[0, T]$ and $v(x, y, t)=v_{2}=0.1 \quad$ for all $x \in[0.5,1], y \in[0,1], t \in[0, T]$ respectively. We also assuming that air pollutant sources are averaged by $Q=0.0001$.The considered air pollutant diffusion coefficient is $D_{x y}=0.1$. The potential air pollutant concentration, $f(x, y)=x(1-x) y(1-y)$. There is no rate of change of air pollutant concentration at all boundaries. By using the Eq. (3.8), and Eqs. (3.14-3.17), we can obtain the approximated air pollutant concentration along the considered street canyon in Fig.3(a), Table 1 and Fig. 4.

Table 1. The approximated air pollutant concentration $c(x, y, 0.1)$

\begin{tabular}{cccccc}
\hline$y / x$ & 0 & 25 & 50 & 75 & 100 \\
\hline 0 & 0.107635 & 0.115472 & 0.121289 & 0.116280 & 0.108312 \\
25 & 0.115035 & 0.129396 & 0.140440 & 0.130930 & 0.116282 \\
50 & 0.119792 & 0.138688 & 0.153221 & 0.140706 & 0.121432 \\
75 & 0.115035 & 0.129396 & 0.140440 & 0.130930 & 0.116282 \\
100 & 0.107635 & 0.115472 & 0.121289 & 0.116280 & 0.108312 \\
\hline
\end{tabular}




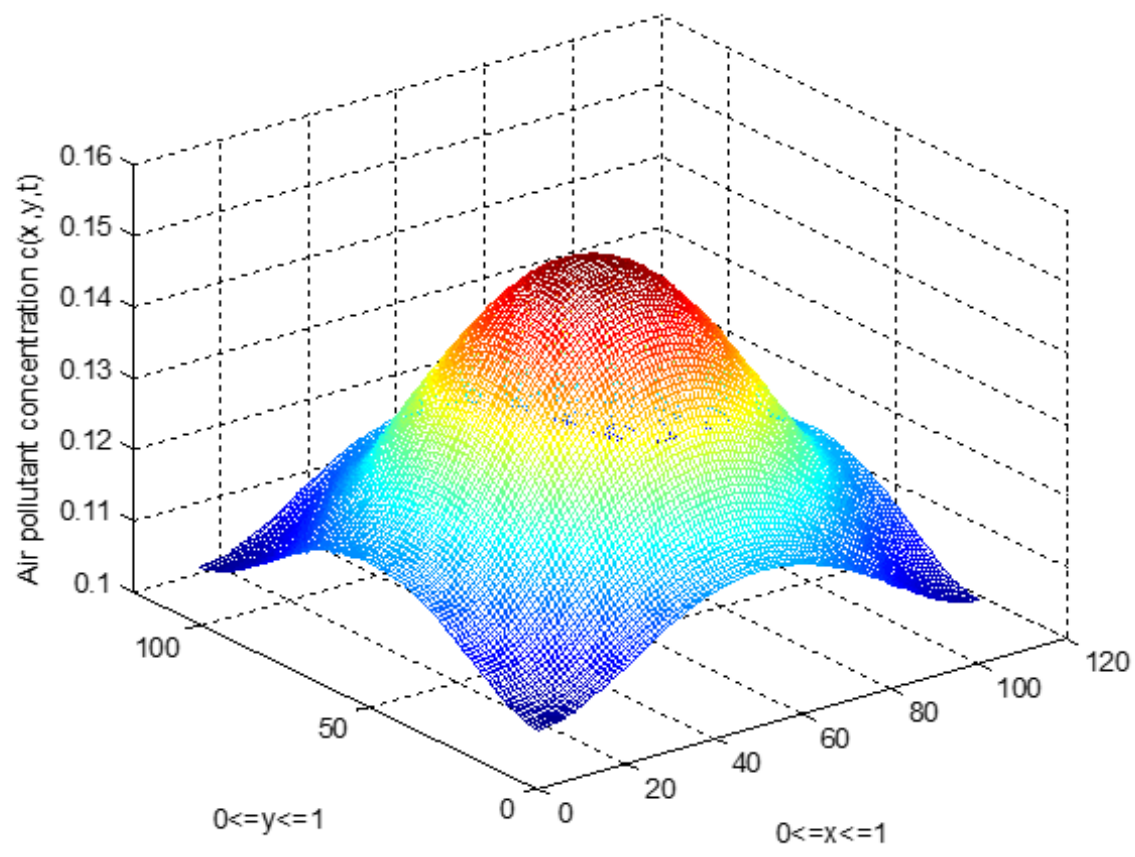

Figure 4. The approximated air pollutant concentration $c(x, y, 0.1)$ for Simulation 1

\subsection{Simulation 2}

Smoothly air flow along a street canyon. Assuming that the averaged air flow velocity in $\mathrm{x}$-and $\mathrm{y}$-direction are constants, $u(x, y, t)=\left(2-x^{2}\right)+\left(2-y^{2}\right)$, for all $(x, y, t) \in \Omega, v(x, y, t)=v=0.1$ for all $(x, y, t) \in \Omega$. We also assuming that air pollutant sources are interpolated by $Q=\left(0.2133 \times 10^{-4}\right) x^{3}+\left(0.22 \times 10^{-4}\right) x^{2}$.

$$
-\left(0.3633 \times 10^{-4}\right) x+\left(1.2041 \times 10^{-4}\right)
$$

The considered air pollutant diffusion coefficient is $D_{x y}=0.1$. The potential air pollutant concentration, $f(x, y)=x(1-x) y(1-y)$. There is no rate of change of air pollutant concentration at all boundaries. There is air pollutant measurement $c(0, y, t)=1$ along the entrance. By using Eq. (3.8), and Eqs. (3.14-3.17), we can obtain the approximated air pollutant concentration along the considered street canyon in Fig.3 (b), Table 2, and Fig.5.

Table 2. The approximated air pollutant concentration $c(x, y, 0.1)$

\begin{tabular}{cccccc}
\hline$y / x$ & 0 & 25 & 50 & 75 & 100 \\
\hline 0 & 1 & 0.956614 & 0.354858 & 0.135288 & 0.135614 \\
25 & 1 & 0.963941 & 0.374452 & 0.153353 & 0.152844 \\
50 & 1 & 0.942766 & 0.346566 & 0.166201 & 0.165161 \\
75 & 1 & 0.896396 & 0.288386 & 0.153409 & 0.153102 \\
100 & 1 & 0.839355 & 0.234387 & 0.134212 & 0.136109 \\
\hline
\end{tabular}




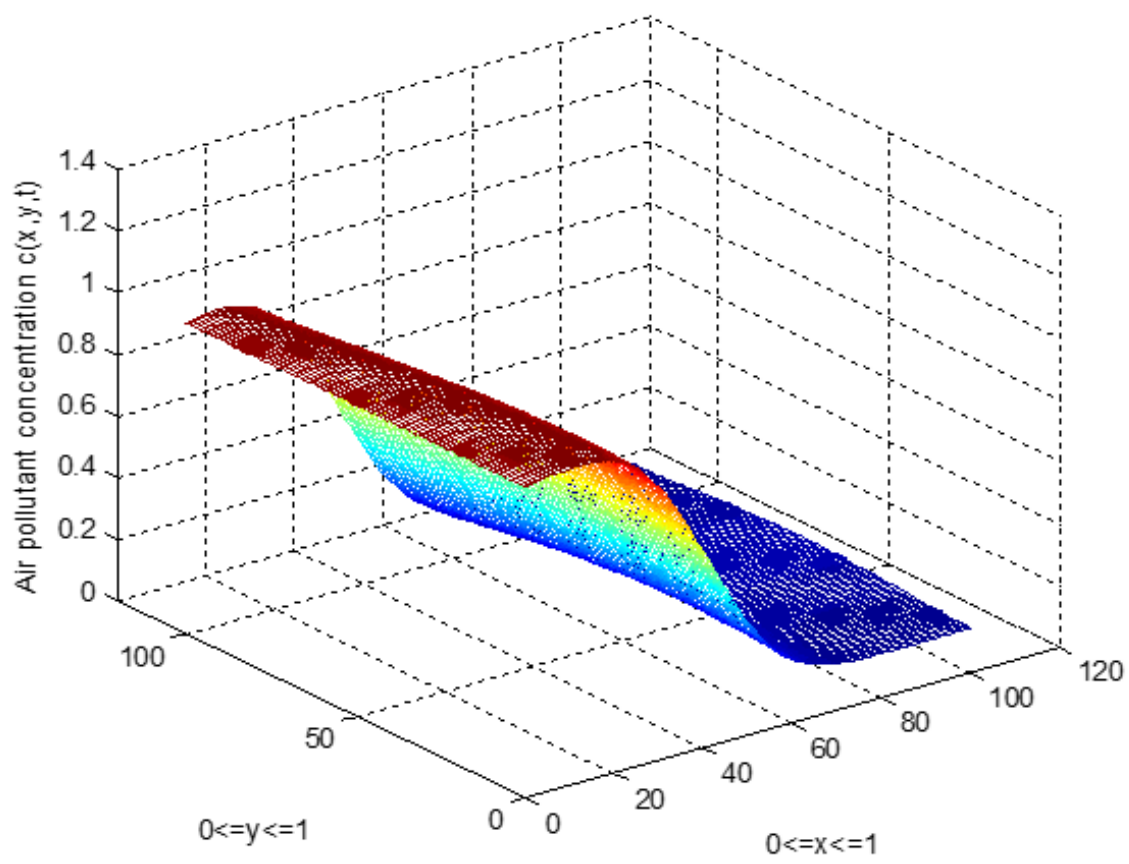

Figure 5. The approximated air pollutant concentration $c(x, y, 0.1)$ for Simulation 2

\subsection{Simulation 3}

Smoothly air flow along a street canyon. Assuming that the averaged air flow velocity in $\mathrm{x}$-and $\mathrm{y}$-direction are constants, $u(x, y, t)=\left(2-x^{2}\right)+\left(2-y^{2}\right)$, for all $(x, y, t) \in \Omega, v(x, y, t)=v=0.1$ for all $(x, y, t) \in \Omega$. We also assuming that air pollutant sources are interpolated by $Q=\left(0.2133 \times 10^{-4}\right) x^{3}+\left(0.22 \times 10^{-4}\right) x^{2}$. $-\left(0.3633 \times 10^{-4}\right) x+\left(1.2041 \times 10^{-4}\right)$

The considered air pollutant diffusion coefficient is $D_{x y}=0.1$. The potential air pollutant concentration, $f(x, y)=x(1-x) y(1-y)$. There is no rate of change of air pollutant concentration at all boundaries. By using the Eq. (3.8), and Eqs. (3.14-3.17), we can obtain the approximated air pollutant concentration along the considered street canyon in Fig.3 (b), Table 3, and Fig.6.

Table 3. The approximated air pollutant concentration $c(x, y, 0.1)$

\begin{tabular}{cccccc}
\hline$y / x$ & 0 & 25 & 50 & 75 & 100 \\
\hline 0 & 0.122374 & 0.120109 & 0.121968 & 0.130561 & 0.135608 \\
25 & 0.125118 & 0.122543 & 0.130588 & 0.148125 & 0.152838 \\
50 & 0.126537 & 0.124571 & 0.139030 & 0.162520 & 0.165158 \\
75 & 0.125510 & 0.123507 & 0.135012 & 0.151458 & 0.153100 \\
100 & 0.122693 & 0.120797 & 0.1254503 & 0.133248 & 0.136108 \\
\hline
\end{tabular}




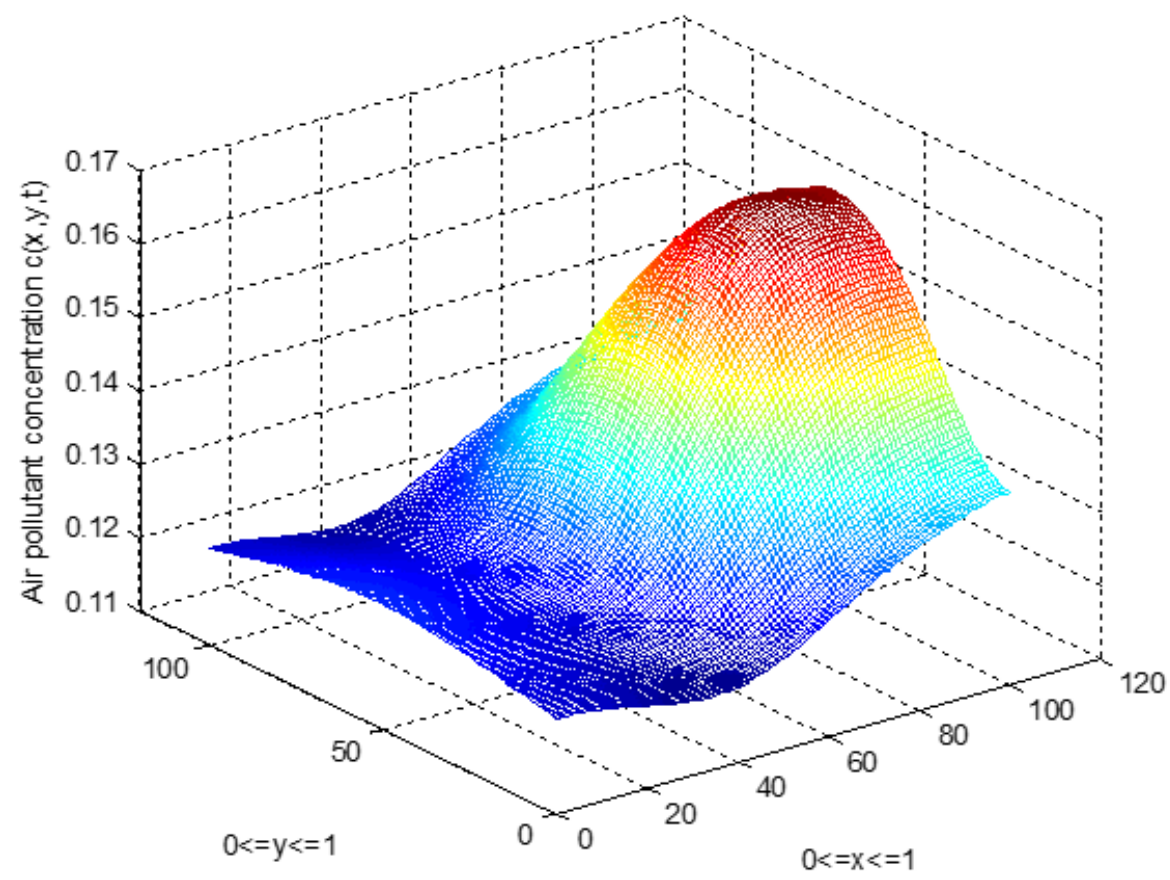

Figure 6. The approximated air pollutant concentration $c(x, y, 0.1)$ for Simulation 3

\section{Discussion}

When the average air pollutant source is static, as indicated in Table 1 and Fig. 4, the total air pollutant concentration level will grow along a given street canyon. It indicates that if there is no rate of change in air pollutant concentration at the entrance and departure gates, the controlled air pollutant source has a low effect on operating. As demonstrated in Table 2 and Fig.5, the external air pollution source has an impact on the computed air pollutant level and its increasing in simulation 2. An interpolation function can be created using field data on air pollution concentrations so as to get an implementation of the model. In simulation 3, the interpolation functions for collected air flow data and interior obtained air pollution concentration data are integrated. As indicated in Table 3 and Fig.6, air pollutants will flow through the canyon depending on the air flow direction.

\section{Conclusions}

A two-dimensional vertically averaged air pollution model in a street canyon is solved using the finite difference method. Several finite difference techniques are utilized at the entry, north, south, and exit of an air pollution model in a street canyon, depending on a good approximation. The introduced computational algorithms result in an effective concentration of air pollution.

\section{Acknowledgments}

This paper is supported by the Centre of Excellence in Mathematics, the Commission on Higher Education (CEM) Thailand.

\section{REFERENCES}

[1] Antoine P.R.J, Riccardo B., James E., and Paul S.M. 2017. "Air quality affected by trees in real street canyons: The case of Marylebone neighbourhood in central London." Urban Forestry \& Urban Greening. 22, 41-53.

[2] Konglok, S.A. and Pochai, N. 2012. "A Numerical Treatment of Smoke Dispersion Model from Three Sources using Fractional Step Method." Adv. Studies. Theor. Phys. 6(5): 217-223.

[3] Konglok, S.A. and Tangmanee, S. 2002. "Numerical Solution of Advection-Diffusion of an Air Pollutant by the Fractional Step Method." Proceeding in the 3rd national symposium on graduate research. Nakonratchasrima Thailand, 18th-19th July 2002.

[4] Konglok, S.A., and Pochai, N. 2016. "Numerical Computations of Three-dimensional Air Quality Model with Variations on Atmospheric Stability Classes and Wind Velocities using Fractional Step Method." IAENG International Journal of Applied Mathematics. 46, 112-120.

[5] Konglok, S.A., Pochai, N., Tangmanee, S. 2009. "A Numerical Treatment of the Mathematical Model for Smoke Dispersion from Two Sources." Proceeding in ICMA-MU 2009. 4, 199-202. 
[6] Konglok, S.A., Tangmanee, S. 2007. "A K-model for Simulating the Dispersion of Sulfur Dioxide in a Tropical Area." Journal of Interdisciplinary Mathematics. 10, 789-799.

[7] Laura E.V., Nicolás A.M., and Mariana C.D. 2014. “A simple model for calculating air pollution within street canyons.” Atmospheric Environment. 87, 77-86

[8] Olga V.T., Sergey V.M., Anne P., Anatoly A.L., Yuri V.Z. 2009. "Air pollution dispersion within urban street canyons." Atmospheric Environment. 43, 245-252 